INPLASY

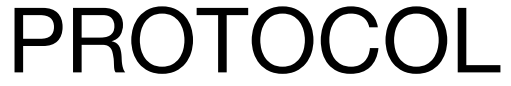

To cite: Yang. Prognostic Value

Of Sparc In Hepatocellular

Carcinoma. Inplasy protocol

202180115. doi:

10.37766/inplasy2021.8.0115

Received: 30 August 2021

Published: 30 August 2021

Corresponding author:

Yunhong Xia

xy_yang211@126.com

Author Affiliation:

Department of Oncology, the

Fourth Affiliated Hospital,

Anhui MedicalUniversity.

Support: NNSF of China, No.81472331.

Review Stage at time of this submission: Piloting of the study selection process.

\section{Prognostic Value Of Sparc In Hepatocellular Carcinoma}

Conflicts of interest:

None declared.

Review question / Objective: Population: patients with hepatocellular carcinoma. Intervention: qPCR,WB or IHC to test SPARC level. Control: peritumoral tissue and HCC with low SPARC level. Outcomes: OS. Study design: systematic review and meta-analysis.

Condition being studied: Focusing on the relatives between SPARC level in patients with hepatocellular carcinoma and their survival outcome, we compare them with data from peritumoral tissue and patients with low SPARC level.

Information sources: Databases: Embase, Pubmed, Web of Science, Wanfang, CBM, CNKI.

INPLASY registration number: This protocol was registered with the International Platform of Registered Systematic Review and Meta-Analysis Protocols (INPLASY) on 30 August 2021 and was last updated on 30 August 2021 (registration number INPLASY202180115).

\section{INTRODUCTION}

Review question / Objective: Population: patients with hepatocellular carcinoma. Intervention: qPCR,WB or IHC to test SPARC level. Control: peritumoral tissue and HCC with low SPARC level. Outcomes:
OS. Study design: systematic review and meta-analysis.

Condition being studied: Focusing on the relatives between SPARC level in patients with hepatocellular carcinoma and their survival outcome, we compare them with 
data from peritumoral tissue and patients with low SPARC level.

\section{METHODS}

Search strategy: ('sparc' OR 'osteonectin' OR 'secreted protein acidic and cysteine rich') AND ('hcc' OR 'hepatic stellate cell' OR 'hepatoma' OR 'liver cancer') AND ('survival' OR 'prognosis' OR 'prognostic') databases: Embase, Pubmed, Web of Science, Wanfang, CBM, CNKI.

Participant or population: Patients with hepatocellular carcinoma.

Intervention: No.

Comparator: SPARC level, through PCR/ Western Blotting/IHC.

Study designs to be included: Studies reported the association between SPARC expression and survival outcome.

Eligibility criteria: (a) clinical studies researched patients with hepatocellular carcinoma; (b) SPARC expression in hepatocellular carcinoma was measured with methods such as immunohistochemistry (IHC) or Quantitative realtime polymerase chain reaction (qRT-PCR) or Western Blotting(WB); (c) studies reported the association between SPARC expression and survival outcome; (d) studies contained $\mathrm{HRs}$ and $95 \% \mathrm{Cl}$ for OS according to SPARC status which either were reported or could be estimated from the relevant published data(e) only the most recent report or the most integrated report would be enrolled, if the study population was duplicated or overlapping.

Information sources: Databases: Embase, Pubmed, Web of Science, Wanfang, CBM, CNKI.

Main outcome(s): The association between SPARC expression and survival outcome.

Quality assessment / Risk of bias analysis: Not reported.

Strategy of data synthesis: Not reported.
Subgroup analysis: Not reported.

Sensitivity analysis: Not reported.

Language: English.

Country(ies) involved: China.

Keywords: HCC; SPARC; prognosis.

Contributions of each author:

Author 1 - Xiaoyu Yang. 